\title{
Novel synthesis of porous carbons with tunable pore size by surfactant-templated sol-gel process and carbonisation
}

\author{
Kyu Tae Lee and Seung M. Oh* \\ School of Chemical Engineering and Research Centre for Energy Conversion \& Storage, Seoul National \\ University, Seoul 151-744, Korea.E-mail: seungoh@plaza.snu.ac.kr; Fax: 82-2-872-5755; \\ Tel: $82-2-880-7074$
}

Received (in Cambridge, UK) 16th August 2002, Accepted 4th October 2002

First published as an Advance Article on the web 21st October 2002

Surfactant-templated sol-gel polymerisation was explored to synthesize the resorcinol-formaldehyde (RF) gels without supercritical drying step, which were further carbonised to obtain porous carbons of a tunable pore size.

Since Pekala reported the preparation of resorcinol-formaldehyde (RF) aerogels by sol-gel polymerisation and subsequent supercritical drying, ${ }^{1} \mathrm{RF}$ aerogels have been widely explored as an intermediate for mesoporous carbons. ${ }^{2}$ Even if the aerogelderived carbons have a high surface area and large mesopore volume, thereby applicable to adsorbents, electric double-layer capacitors, and materials for chromatographic separation, ${ }^{3}$ the cost of supercritical drying is extremely expensive. As an alternative less-expensive preparation method, solvent exchange or freeze-drying has been proposed. ${ }^{4}$ Here, we report another low-cost preparation method for RF xerogels, where a normal drying is still applicable because the pore collapse can be minimized during the water evaporation. The resulting RF xerogels are carbonised to obtain porous carbons with tunable pore size from macropores to mesopores.

The novel feature in this synthesis is the utilization of surfactant-template in the sol-gel polymerisation reaction. The surfactant molecules play two important roles in this synthesis. Firstly, they form spherical micelles in water and act as a template for the sol-gel polymerisation. The micro- to nanosized spherical gel clusters are formed within the micelles, which are further three-dimensionally connected via a crosslinking reaction. As a result, interstitial sites are generated that become pores. Secondly, the surfactant molecules still adsorb on the pore walls even after the polymerisation reaction, such that they eventually lower the surface tension at the pore waterresin interface. This minimizes the pore collapse during the drying step.

The synthetic procedure is shown in Fig. 1. In detail, the surfactant (cetyltrimethylammonium bromide, CTAB) is dispersed in deionised water. Then, the mixture of resorcinol (R), formaldehyde $(\mathrm{F})$, and sodium carbonate (as a catalyst) is added to obtain surfactant-stabilized (templated) RF sols. Heating at $85{ }^{\circ} \mathrm{C}$ induces a polymerisation reaction inside the surfactanttemplated RF sols to generate spherical gel clusters. An interconnection between the RF gel clusters is also possible because their surface is still active for the cross-linking reaction.
The resulting RF gels are dried at $85^{\circ} \mathrm{C}$ and further heat-treated at $1000{ }^{\circ} \mathrm{C}$ for $3 \mathrm{~h}$ under argon atmosphere to obtain porous carbons in monolithic shape.

Table 1 lists the synthetic conditions and physical properties of the porous carbons. The RF gel prepared without the use of surfactant-template carries a negligible pore structure and thus the resulting carbon $(\mathrm{C} 1)$ shows a specific surface area of $<1$ $\mathrm{m}^{2} \mathrm{~g}^{-1}$. This is the result of pore collapse during the drying step due to large capillary forces imposed at the liquid-vapor interface. ${ }^{5}$ Interestingly, however, when the surfactant-template is used, the RF xerogels exhibit a rather stable pore structure even after a normal drying. This feature must arise from the reduction of surface tension by the adsorbed surfactant molecules. The resulting carbons show a well-developed pore structure as described below.

Fig. 2 shows the field-emission scanning electron microscopy (FE-SEM) images of one RF gel and three porous carbons. The spherical gel clusters and pores made at the interstitial sites can be recognized in Fig. 2(a). In this particular synthesis, the size of the gel clusters is $c a$. $100 \mathrm{~nm}$ (Fig. 2(a)), but reduced to ca. $65 \mathrm{~nm}$ by carbonisation (Fig. 2(b)). The pores made by the
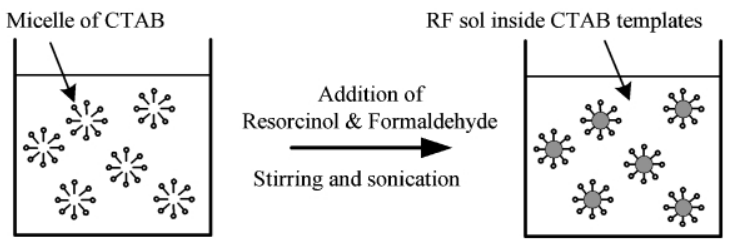

Polymerisation at $85^{\circ} \mathrm{C}$
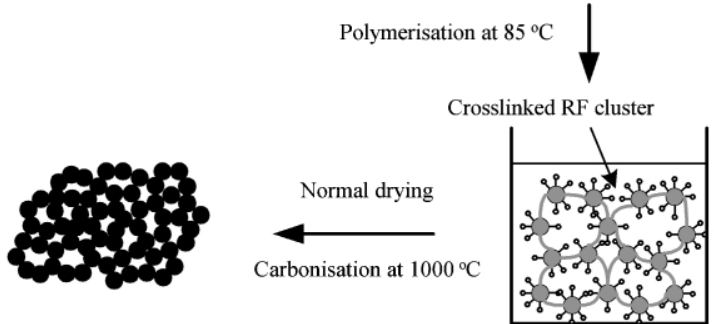

Fig. 1 The synthetic scheme for porous carbons. The detailed explanation is given in the text.

Table 1 Synthetic conditions, relative molar ratios of reagents and physical properties of porous carbons

\begin{tabular}{|c|c|c|c|c|c|c|c|c|c|}
\hline Sample & $\mathrm{R} / \mathrm{mol}$ & $\mathrm{F} / \mathrm{mol}$ & $\mathrm{CTAB} / \mathrm{mol}$ & $\mathrm{H}_{2} \mathrm{O} / \mathrm{mol}$ & $\mathrm{pH}$ & $\begin{array}{l}\text { Surface area } / \mathrm{m}^{2} \\
\mathrm{~g}^{-1}\end{array}$ & $\begin{array}{l}\text { Cluster diameter }{ }^{a} \text { } \\
\mathrm{nm}\end{array}$ & $\begin{array}{l}\text { Pore diameter } b / \\
\mathrm{nm}\end{array}$ & $\begin{array}{l}\text { Pore volume } \\
\mathrm{cm}^{3} \mathrm{~g}^{-1}\end{array}$ \\
\hline $\mathrm{C} 1$ & 1 & 2 & 0 & 5.62 & 6.3 & $<1$ & - & - & - \\
\hline $\mathrm{C} 2$ & 1 & 2 & 0.082 & 5.62 & 5.0 & 10 & ca. 2000 & - & - \\
\hline C3 & 1 & 2 & 0.082 & $5.62 \times 20$ & 6.0 & 426 & ca. 160 & $>60$ & 0.40 \\
\hline $\mathrm{C} 4$ & 1 & 2 & 0.082 & $5.62 \times 30$ & 6.0 & 422 & ca. 65 & 31 & 0.51 \\
\hline $\mathrm{C} 5$ & 1 & 2 & 0.082 & $5.62 \times 10^{3}$ & 7.1 & 419 & ca. 15 & 3.9 & 0.24 \\
\hline C6 & 1 & 50 & 0.082 & $5.62 \times 10^{3}$ & 6.7 & 461 & ca. 15 & 6.5 & 0.35 \\
\hline C7 & 1 & 100 & 0.082 & $5.62 \times 10^{3}$ & 6.4 & 501 & ca. 15 & 7.4 & 0.41 \\
\hline
\end{tabular}

diameter was assessed from the FE-SEM and TEM images. ${ }^{b}$ Pore diameter is the peak value in pore size distribution that was calculated by the 
spherical carbon clusters are $31 \mathrm{~nm}$ in diameter (Fig. 2(b)) and the Brunauer-Emmett-Teller (BET) surface area is $422 \mathrm{~m}^{2}$ $\mathrm{g}^{-1}$.

In this preparation, the pore size is primarily determined by the size of gel clusters, which is in turn controlled by the monomer/surfactant concentration. As the micellar size becomes smaller, the size of gel clusters and thus the carbon pore size decrease with a dilution of the monomer/surfactant content (Fig. 2(b)-(d)).

The sol-gel polymerisation of resorcinol (R) with formaldehyde $(\mathrm{F})$ is composed of two consecutive reactions: addition and condensation with the latter acid-catalysed to produce a cross-linked polymer network. ${ }^{4}$ It is thus very likely that, in acidic conditions, the cross-linking between gel clusters becomes more facilitated to produce a more rigid gel network, and the pore collapse or shrinking at both the drying step and subsequent carbonisation step is suppressed. To test this possibility, the sol-gel polymerisation was performed at different $\mathrm{pH}$ by varying the $\mathrm{R} / \mathrm{F}$ ratio in this work (samples $\mathrm{C5}$ C7). As listed in Table 1, the sol-gel reaction medium becomes more acidic within the $\mathrm{pH}$ range of 7.1-6.4 with an increase in the formaldehyde content. When the pore properties are compared for samples $\mathrm{C} 5-\mathrm{C} 7$, there appears a strong correlation between the porosity and $\mathrm{pH}$ of the sol-gel medium even if the carbon cluster size is comparable for the three because the monomer/surfactant concentration is the same. The RF gels and carbons prepared under more acidic conditions exhibit larger surface areas, pore diameters and pore volumes. This must be the result of rigidity enhancement by the acid-catalysed crosslinking reaction between the gel clusters.

Fig. 3(a) presents the nitrogen adsorption and desorption isotherm for sample $\mathrm{C} 7$. This porous carbon as well as the others shows a type IV isotherm with a hysteresis loop that is associated with capillary condensation in mesopores and with a
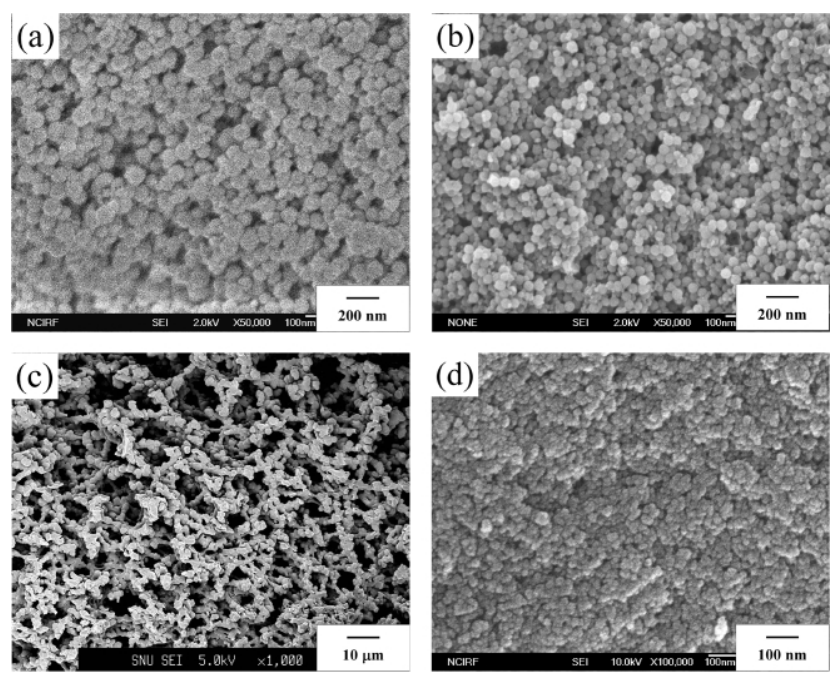

Fig. 2 FE-SEM images of porous materials: (a) the RF precursor gel for $\mathrm{C}$ before carbonisation; (b) C4; (c) C2; (d) C5. The images were obtained with a JEOL JSM 6700F and 6330F. Note the uniform-sized spherical clusters and pores formed at the interstitial sites.
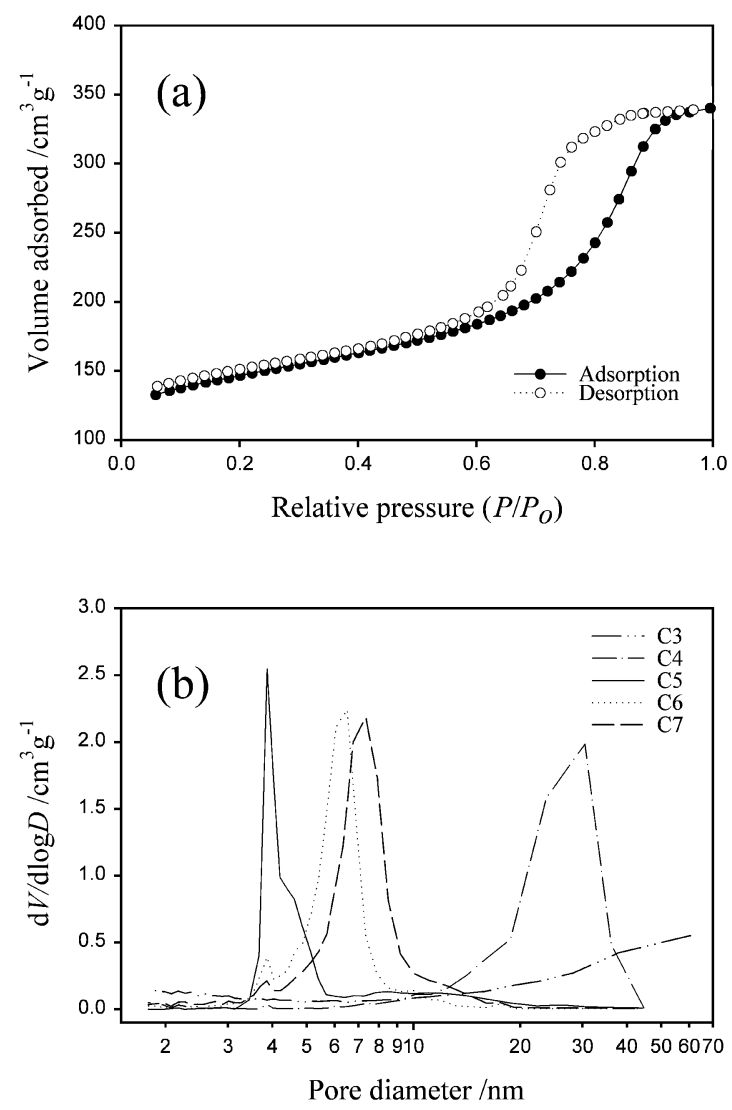

Fig. 3 (a) The $\mathrm{N}_{2}$ adsorption-desorption isotherm of mesoporous carbon (C7). (b) The pore size distribution of C3 (dash-dot-dot line), C4 (dash-do line), C5 (solid line), C6 (dotted line) and C7 (dashed line) calculated from the desorption branch of the nitrogen isotherm. The isotherms were measured at $77 \mathrm{~K}$ with a Micrometrics ASAP2000 Gas Adsorption Analyser.

plateau at high relative pressure, indicating the dominance of mesopores in these samples. Fig. 3(b) shows the pore size distribution of samples C3-C7 as calculated by the BarrettJoyner-Halenda (BJH) method from the desorption branch of the isotherm. It is seen that the pore size is uniform in each sample and the size can be controllable within the mesopore range.

\section{Notes and references}

1 R. W. Pekala, J. Mater. Sci., 1989, 24, 3221

2 R. Saliger, V. Bock, R. Petricevic, T. Tillotson, S. Geis and J. Fricke, J. Non-Cryst. Solids, 1997, 221, 144; A. W. P. Fung, Z. H. Wang, K. Lu, M. S. Dresselhaus and R. W. Pekala, J. Mater. Res., 1993, 8, 1875; S. Han and T. Hyeon, Chem. Commun., 1999, 1955.

3 R. Ryoo, S. H. Joo, M. Kruck and M. Jaroniec, Adv. Mater., 2001, 13, 677.

4 C. Lin and J. A. Ritter, Carbon, 1997, 35, 1271; H. Tamon, H. Ishizaka, T. Yamamoto and T. Suziki, Carbon, 1999, 37, 2049.

5 C. J. Brinker and G. W. Scherer, J. Non-Cryst. Solids, 1985, 70, 301 